\title{
Encuesta de Salud SF-36: Validación en Tres Contextos Culturales de México
}

\author{
Health Survey SF-36: Validation in Three Cultural Contexts of Mexico
}

\author{
Rozzana Sánchez Aragón ${ }^{1}$, Melissa García Meraz ${ }^{2}$ y Bertha Dolores Martínez Trujillo ${ }^{3}$
}

\section{Resumen}

La presente investigación estuvo orientada a la validación de la Encuesta de Salud SF-36 en tres contextos socio-culturales de México (Ciudad de México, Pachuca, Hidalgo y Gómez Palacio, Durango). Los hallazgos mostraron por un lado, similitudes entre las tres ciudades o entre dos en algunos de los factores observándose también diferencias particulares a los ambientes. Por otro lado, los factores obtenidos en las muestras de esta investigación mostraron validez de constructo con la versión original aplicada a una muestra mexicana del sureste (Zúñiga et al., 1999) y coeficientes de confiabilidad Alpha de Cronbach similares a las aplicaciones a diversas muestras españolas.

Palabras clave: salud, física, mental, medición, validación

\begin{abstract}
This research was oriented to the validation of the Health Survey SF-36 in three socio-cultural contexts in Mexico (Mexico City, Pachuca, Hidalgo \& Gómez Palacio, Durango). The findings showed -on one handsimilarities among the three cities or between two of them in some of the factors obtained, and we observed algo, some particular differences in those environments. On the other hand, the obtained factors in the samples of this study displayed construct validity with the original version applied to southern mexican sample (Zúñiga et al., 1999) and their alpha realiability coeficients were similar to the applications made in spanish samples.
\end{abstract}

Keywords: health, physical, mental, measurement, validation

Proyecto Financiado por la Dirección General de Apoyo al Personal Académico IN301814. Efectos de la calidad relacional y la coregulación emocional en la salud física y mental de parejas mexicanas y brasileñas

1 Doctorado. Universidad Nacional Autónoma de México. Profesora Titular. Av. Universidad 2004, Col. CopilcoUniversidad, C. P. 04510, Del Coyoacán, México, D. F. Tel. : 56228222 ext. 41223. Correo: rozzara@unam.mx

${ }^{2}$ Doctorado. Universidad Autónoma del Estado de Hidalgo. Profesora. Privada del Balcón 103 Col. Haciendas de Hidalgo, C.P. 42082, Pachuca, Hgo. Mexico. Tel.: 5544434734. Correo: melissaunam@ yahoo.com.mx

${ }^{3}$ Maestría. Universidad Juárez del Estado de Durango. Profesora. Calzada Palmas I y Calle Sixto Ugalde, C. P. 35050 ,

Gómez Palacio, Dgo. Tel.: (871) 7146467 Correo: bedolmart@gmail.com 


\section{Introducción}

La salud es un tema de controversia, ya que hay muchos aspectos que se deben tomar en cuenta para poder medirla por completo. Para la Organización Mundial de la Salud (OMS), la salud es el estado completo de bienestar físico psicológico y social que tiene una persona. Para Cruceanu, Muntele y Cozma (2014) es la conexión entre el bienestar funcional y la capacidad del cuerpo para adaptarse a las diferentes áreas de la vida humana, ya sea laboral, intelectual, personal o social. Toda persona requiere de un bienestar objetivo y otro subjetivo, este último por lo general se conecta con la calidad de vida (CV) que abarca muchos aspectos medibles del ser humano, los cuales son evaluados de acuerdo a su actitud ante situaciones adversas, satisfaccion ante su persona y hacia su vida. Por lo tanto, las personas perciben su CV de acuerdo a su historia personal, y juega un papel importante en la comprension de sus mecanismos y vinculación con su estado de salud, su prosperidad y su bienestar en general (Fleuret \& Thouez, 2007).

$\mathrm{La} C \mathrm{C}$ en general se refiere a una propiedad que tiene el individuo para experimentar situaciones y condiciones de su ambiente, dependiendo de las interpretaciones y valoraciones que hace de los aspectos objetivos de su entorno. Asimismo Ardila (2003) considera que es un estado de satisfacción general, derivado de la realización de las potencialidades de la persona que poseé aspectos subjetivos (sensación subjetiva de bienestar físico, psicológico y social, la intimidad, la expresión emocional, la seguridad percibida, la productividad personal) y aspectos objetivos (bienestar material, las relaciones armónicas con el ambiente físico y social y con la comunidad, y la salud objetivamente percibida).

Conceptos como CV o calidad de vida relacionada con la salud (CVS), permiten estudiar $\mathrm{y}$ evaluar el estado de salubridad como un predictor de CV personal, lo cual, se convierte en la unidad fundamental para la medición de resultados en investigaciones dentro de este campo. Aunque no exista consenso, la CVS se mide por medio de la función física, del estado psicológico, de la función y la interacción social y de los síntomas físicos, todos ellos elementos del espectro de indicadores para la medición de 5 dominios principales relacionados con la salud: mortalidad, morbilidad, incapacidad, incomodidad e insatisfacción (Shmueli, 2002).

En la búsqueda por la medición del constructo, el Cuestionario de Salud SF-36 toma en cuenta la percepción del individuo respecto a su CVS y así obtener un perfil de éste que refleja dimensiones no solo clínicas del concepto de enfermedad, sino un completo bienestar físico, psíquico y social. Por lo tanto esta medida es un elemento importante en los estudios sobre el estado de salud y sus determinantes y juega un papel de interés en la comprensión de los mecanismos que son la base de la percepción del estado de salud, de la prosperidad y del bienestar en general.

La CVS comprende las dimensiones de incapacidad, incomodidad e insatisfacción. La primera se examina en relación con la habilidad para realizar actividades y por el impacto físico que las mismas producen en la esfera física, ocupacional y cotidiana. La incomodidad refiere a la presencia o ausencia del dolor físico y la fatiga. Finalmente, la insatisfacción corresponde con el funcionamiento social, el bienestar general y el grado de satisfacción con la atención que brindan los prestadores de servicios de salud (Zúñiga et al., 1999). La medida señalada reconoce al individuo como un ser bio-psico-social, en el que se reconocen diferentes determinantes, que influyen en el estado de salud general. En lo biológico se pueden encontrar factores individuales como la presencia o ausencia de enfermedad, en lo psicológico se identifica la determinación del estado de salud mental como el optimismo y la personalidad, y en lo social se aborda la dinámica interaccional con la familia, amigos, pareja, trabajo, etc.

Inicialmente, este instrumento se usó en el estudio de resultados médicos, donde se demostró su validez y confiabilidad; asimismo, se determinaron las normas de comparación para la población de Estados Unidos de América. Posteriormente, el proyecto IQOLA (International Quality of Life Assessment Project) incluyó 14 países industrializados, y en la actualidad hay más de 40 naciones participantes. Existen seis versiones de la encuesta SF-36 en español que se han adaptado y utilizado en Argentina, Colombia, España, Honduras y México, así como entre la 
población México-Estadounidense. El SF-36 se ha utilizado en investigaciones clínicas especificas; por ejemplo, Vilagut et al., (2005) informaron sobre la validez y la confiabilidad de una versión utilizada en España, a partir de un estudio realizado con 46 pacientes con cardiopatía coronaria estable. Por su parte, Zúñiga, CarrilloJiménez, Fos, Gandek y Medina-Moreno (1999) utilizaron la versión 1.1 de la encuesta, autorizada para usarse en México con el fin de medir el estado funcional de una muestra de individuos del sureste del país.

En la actualidad hay un considerable incremento en la literatura médica respecto de la inclusión de la evaluación que los pacientes tienen sobre su estado de salud y la incorporación de la CV con la salud informada por el paciente, de acuerdo a su vida cotidiana, lo que resulta fundamental para poder estimar los resultados clínicos (Guyatt, Kirshner, \& Jaeschke, 1992). De este modo, el cuestionario SF-36 se torna en una herramienta clínica y de investigación, cuyos reactivos y opciones de respuesta ofrecen una redacción entendible y puntuaciones que reflejan la individualidad de cada elemento influyente de la salud del paciente, diferencias especificas de la enfermedad y una autoevaluación muy eficaz del estado de salud. Sobre la base de estas consideraciones, el propósito de la presente investigación fue la validación en el sureste de México, en tres contextos culturales distintos (Ciudad de México, Ciudad de Pachuca, Hidalgo y Ciudad de Gómez Palacio, Durango) de dicha medida e identificar las similitudes y/o diferencias en su configuración factorial.

\section{Método}

\section{Participantes}

Se trabajó con una muestra no probabilística propositiva (Kerlinger \& Lee, 2002) de 460 habitantes de la Ciudad de México, de los cuales 220 eran hombres y 237 mujeres, cuyas edades oscilaron entre los 19 y 76 años $(M=39.86$, $D E=12.67)$. De la muestra total, 316 señalaron estar casados, y 138 mencionan vivir en unión libre. En cuanto a la escolaridad, 89 participantes tenían estudios de secundaria, 235 de preparatoria y 120 de licenciatura y 2 de posgrado. Todos los participantes informaron tener una relación de pareja en el momento de participar en el estudio, de un mínimo de 12 meses y hasta 648 meses $(\mathrm{M}=189.01$ meses, $\mathrm{DE}=130.58)$. En cuanto al número de hijos variaba de 0 a 5 .

Se trabajó con una segunda muestra no probabilística propositiva (Kerlinger \& Lee, 2002) de 230 habitantes de la Ciudad de Pachuca, Hidalgo, de los cuales 112 eran hombres y 118 mujeres, cuyas edades oscilaron entre los 17 y 62 años $(M=28.06, D E=10.00)$. De la muestra total, 121 señalaron estar casados, y 109 mencionan vivir en unión libre. En cuanto a la escolaridad, 114 participantes tenían estudios de preparatoria y 116 de licenciatura. Todos ellos informaron tener una relación de pareja, de un mínimo 12 meses hasta 648 meses ( $M=31.87$ meses, $\mathrm{DE}=44.96)$. En relación con el número de hijos, 104 refirieron no tenerlos, 56 inormaron tener dos hijos, 31 tener solo uno, 22 tres, 6 cuatro y 11 cinco.

Se trabajó con una tercera muestra no probabilística propositiva (Kerlinger \& Lee, 2002) de 460 habitantes de la Ciudad de Gómez Palacio, Durango, de los cuales 115 eran hombres y 115 mujeres, cuyas edades oscilaron entre los 18 y 72 años $(M=38.93, D E=11.75)$. De la muestra total, 191 señalaron estar casados, y 39 mencionan vivir en unión libre. En cuanto a la escolaridad, 84 participantes tenían estudios de preparatoria y 146 de licenciatura. Todos los participantes informaron tener una relación de pareja en el momento de participar en el estudio de un mínimo de 12 meses hasta 624 meses $(M=15.49$ meses, $\mathrm{DE}=11.46)$. El número de hijos varió de 0 a 6 .

\section{Instrumento}

Se usó la Escala de Evaluación de la Salud SF-36 (Zúniga et al., 1999) que evalúa aspectos de la calidad de vida en personas mayores de 16 años. Se basa en ocho conceptos de salud: función física, rol físico, dolor corporal, salud general, vitalidad, función social, rol emocional y salud mental. Aunado a estos conceptos incluye el concepto general de cambios en la percepción del estado de salud actual y en la del año anterior. La respuesta a estos cambios describe la transición de la percepción respecto del mejoramiento o empeoramiento del estado de salud. Las definiciones de los conceptos son las siguientes. Salud mental: existencia estable de sentimientos 
de angustia y depresion o de felicidad y tranquilidad. Rol emocional: problemas con el trabajo y otras actividades diarias a consecuencia de problemas emocionales. Funcion Fisica: extensión de las limtaciones de las personas para llevar a cabo todas las actividades fisicas, incluidas bañarse o ducharse, debido a problemas de salud. Rol Físico: problemas con el trabajo u otras actividades diarias que se corresponden con su malestar físico. Dolor corporal: grado de dolencia que limita las actividades realizadas diariamente. Vitalidad: grado de energía con la que cuenta. Salud general: percepción del estado de salud. Función social: frecuencia con la que los problemas físicos o emocionales interfieren con las actividades sociales.

En cuanto a su forma de respuesta la evaluación inicial considera variantes del formato Likert (4 o 5 opciones, en algunos casos utilizando de mala a excelente, de sí me limita mucho a nada en absoluto, de nada a demasiado, de ninguno a muy severo, de siempre a nunca). Otras preguntas admiten respuestas dicotómica cierto/falso y si/no en las diferentes secciones que la componen. Sin embargo, con el fin de poder analizar el mayor número posible de reactivos en un mismo análisis factorial, se unificaron las respuestas de la manera más respetuosa posible en relación con el sentido de las preguntas y con el objetivo de la escala, por lo que se realizó una modificación. Se conservaron algunas secciones como indicadores, ya que incluían uno o cuatro reactivos, como en el caso de salud en general y en la comparación de las percepciones actuales con las de hace un año. Para el resto se homogeneizaron en un formato de cinco puntos, que va de nada a mucho.

La SF-36 es un instrumento auto-aplicado que originalmente contiene 36 preguntas, sin embargo, con el fin de equilibrar los aspectos evaluados y no tener reactivos con dos ideas opuestas se agrgaron 9 reactivos (“A causa de su salud física ¿dejó de hacer por completo sus actividades cotidianas o de trabajo?”; “Puso menos atención a la hora de estar realizando su trabajo o actividades cotidianas debido a algún problema emocional (como estar triste, deprimido o nervioso”?; “Mostró menos interés en convivir con otras personas debido a algún problema emocional (como estar triste, deprimido o nervioso)?”; “HHasta qué punto el dolor le ha dificultado su trabajo habitual (incluido el trabajo fuera de casa y las tareas domésticas)?”; “¿Ha tenido dolor por largos periodos de tiempo?”; “ ¿El dolor físico que ha tenido le ha generado algún problema emocional?”, “¿Realiza sus actividades habituales aunque sienta dolor?” y “¿Qué tanto se sintió débil?”). Cabe señalar que la sección 6 y 10 del SF-36 que exploran respectivamente en qué medida la salud física o problemas emocionales han dificultado las actividades sociales normales con la familia, amigos, vecinos o grupos, y cuánto tiempo la salud física o problemas emocionales han dificultado las actividades sociales (como visitar amigos, parientes, etc.), se separaron en dos reactivos cada uno (uno que explora el impacto de la salud física y otro el de la salud emocional). Con estos reactivos adicionales se logró equilibrar la cantidad por área de salud evaluada. Responder a este instrumento tomó un promedio 15 minutos para completarse.

\section{Procedimiento}

Los cuestionarios fueron aplicados a cada uno de los participantes de las muestras seleccionadas en diferentes lugares como plazas comerciales, domicilios particulares, escuelas, oficinas, etc., en los que se podían encontrar personas con una relación de pareja de mínimo de 12 meses en el momento de la aplicación. Se les solicitó que contestaran en forma voluntaria, anónima y confidencial a la escala en cuestión. Ante la aparición de dudas durante la aplicación se proporcionaron respuestas inmediatas, asimismo se puso a disposición de los participantes sus resultados personales.

\section{Análisis de Datos}

Para realizar los análisis estadísticos pertinentes se trabajó por separado con los reactivos que tenían formas de respuesta distintas (nada a mucho - sección 1 y aquellos con formato totalmente cierta a totalmente falsa - sección 2) y un par de reactivos iniciales ("En general, usted diría que su salud es...” y “¿Cómo diría que es su salud actual comparada con la de hace un año?") se tomaron como indicadores. Con el fin de 
Tabla 1. Análisis Factorial de la Sección 1 de la Encuesta de Salud SF-36, Ciudad de México

\begin{tabular}{|c|c|}
\hline Rol Emocional y Función Social & $\alpha=.93$ \\
\hline $\begin{array}{l}\text { ¿Puso menos atención a la hora de estar realizando su trabajo o actividades cotidianas debido a algún problema } \\
\text { emocional (como estar triste, deprimido o nervioso)?. }\end{array}$ & .812 \\
\hline $\begin{array}{l}\text { ¿No hizo su trabajo o sus actividades cotidianas tan cuidadosamente como de costumbre a causa de algún } \\
\text { problema emocional (como estar triste, deprimido o nervioso)?. }\end{array}$ & .799 \\
\hline $\begin{array}{l}\text { ¿Hizo menos de lo que hubiera querido hacer, a causa de algun problema emocional (como estar triste, } \\
\text { deprimido o nervioso)?. }\end{array}$ & .780 \\
\hline $\begin{array}{l}\text { ¿Mostró menos interés en convivir con otras personas debido a algún problema emocional (como estar triste, } \\
\text { deprimido o nervioso)? }\end{array}$ & .767 \\
\hline $\begin{array}{l}\text { ¿Hasta qué punto sus problemas emocionales han dificultado sus actividades sociales habituales con la familia, } \\
\text { los amigos, los vecinos u otras personas? }\end{array}$ & 679 \\
\hline $\begin{array}{l}\text { ¿Qué tanto sus problemas emocionales le han dificultado sus actividades sociales (como visitar a los amigos o } \\
\text { familiares)?. }\end{array}$ & 679 \\
\hline ¿Qué tanto se sintio tan bajo de moral que nada podía animarle? & .650 \\
\hline $\begin{array}{l}\text { ¿Tuvo que reducir el tiempo dedicado a su trabajo o a sus actividades cotidianas a causa de algùn problema } \\
\text { emocional (como estar triste, deprimido o nervioso)?. }\end{array}$ & .622 \\
\hline ¿Qué tanto se sintió desanimado y triste? & .595 \\
\hline ¿Qué tanto estuvo muy nervioso? & .529 \\
\hline $\begin{array}{l}\text { ¿Qué tanto su salud fisica le ha dificultado realizar sus actividades sociales (como visitar alos amigos o } \\
\text { familiares)?. }\end{array}$ & .474 \\
\hline Media=1.62; DS=.7168 & \\
\hline
\end{tabular}

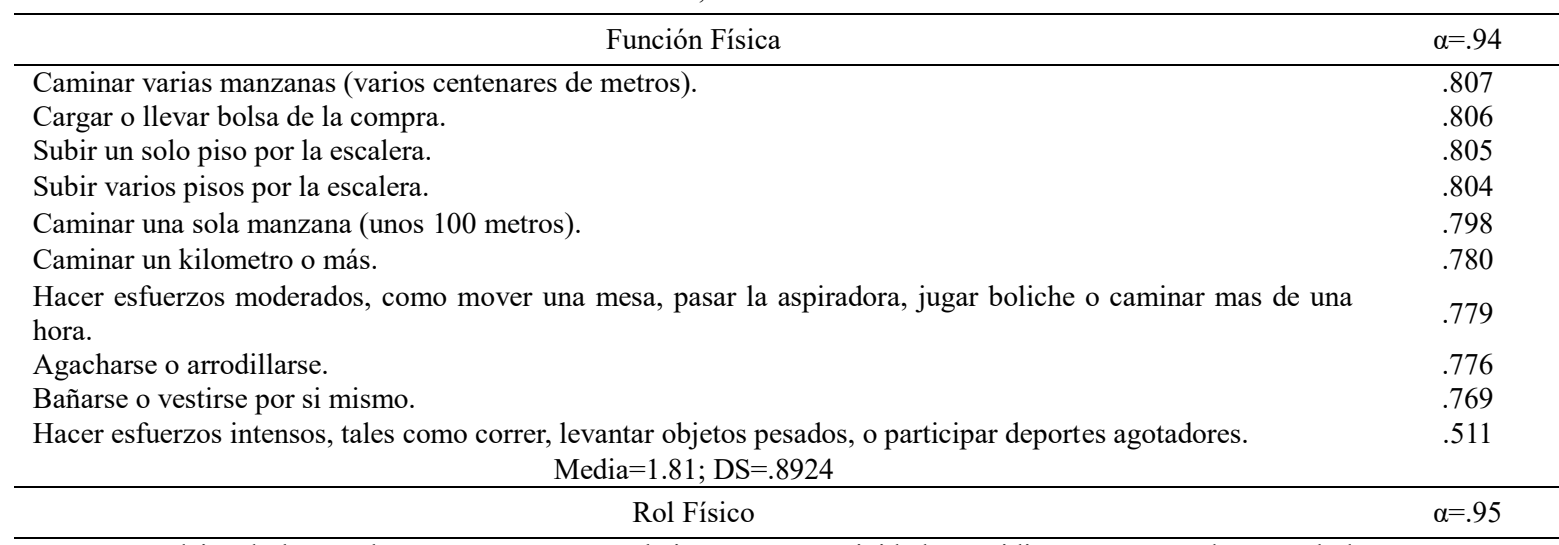

¿Tuvo que dejar de hacer algunas tareas en su trabajo o en sus actividades cotidianas, a causa de sus salud .801

fisica?.
Tuvo alguna dificultad para hacer su trabajo o sus actividades cotidianas (por ejemplo, le costó más de lo normal), a causa de su salud fisica?.

¿Hizo menos de lo que hubiera querido hacer, a causa de su salud fisica?.

Hasta que punto el dolor le ha impedido realizar su trabajo habitual (incluido el trabajo fuera de casa y las tareas doméstica?.

A causa de su salud fisica ¿dejó de hacer por completo sus actividades cotidianas o trabajo?.

¿Hasta que punto el dolor le ha dificultado su trabajo habitual (incluido el trabajo fuera de casa y las tareas doméstica?

¿Tuvo que reducir el tiempo dedicado al trabajo o a sus actividades cotidianas a causa de su salud fisisca?.

¿Hasta que punto su salud fisica ha dificualtado sus actividades sociales habituales con la familia, los amigos, los vecinos u otras personas?.

\begin{tabular}{|c|c|}
\hline ¿Realiza sus actividades habituales aumque sienta dolor?. & .633 \\
\hline ¿Qué tanto se sintió cansado? & .575 \\
\hline ¿Qué tanto se sintió débil? & .564 \\
\hline \multicolumn{2}{|l|}{ Media $=2.09 \mathrm{DS}=.8245$} \\
\hline Vitalidad & $\alpha=.76$ \\
\hline ¿Qué tanto se sintio calmado y tranquilo?. & .788 \\
\hline ¿Qué tanto se sintió lleno de vitalidad?. & .782 \\
\hline ¿Qué tanto tuvo mucha energía?. & .691 \\
\hline
\end{tabular}


obtener una medida válida y confiable se realizaron los siguientes análisis estadísticos para cada sección: 1) se realizó un análisis de frecuencias reactivo por reactivo para obtener media y sesgo; 2) se calculó el estadístico $t$ de Student para muestras independientes (reactivo por reactivo); 3) se realizó un análisis Alpha de Cronbach para evaluar la consistencia interna de la escala con los reactivos que en los análisis anteriores evidenciaron que el sesgo de los ítems se comportaba de acuerdo al tipo de variable estudiada (es decir, si se encontraban dentro de un rango mayor de -.5 a +.5 se consideraban una variable típica o si se encontraban en estos rasgos, la distribución era normal) y mostraron diferencias estadísticamente significativas entre los puntajes altos y bajos de cada reactivo en la prueba $t$; 4) se llevó a cabo un análisis de intercorrelaciones de los reactivos para determinar el tipo de rotación a solicitar en el análisis factorial subsecuente; 5) se realizó un análisis factorial de componentes principales, y 6) el análisis de confiabilidad Alpha de Cronbach por factor.

Los resultados correspondientes a cada una de las ciudades incluidas en el estudio se analizaron una vez concluidos los análisis estadísticos encaminados a identificar los reactivos con mayor poder discriminativo.

\section{Resultados}

\section{Ciudad de México}

En los resultados obtenidos se observó que 44 de los 45 ítems diseñados cumplieron con los criterios establecidos por Reyes Lagunes, García y Barragán (2008). Acto seguido, se analizaron los reactivos de la sección 1 por medio de un análisis factorial de componentes principales con rotación ortogonal (tipo varimax) y se seleccionaron sólo aquellos que registraron cargas factoriales iguales o superiores a .40. El análisis factorial de componentes princiapales arrojó un índice $\mathrm{KMO}=.939$ y la Prueba de Esfericidad de Bartlett=14155.371, gl=780, $p=.000$, además de indicar la existencia de 5 factores con valores superiores a 1 que explicaron el $65.36 \%$ de la varianza y coeficientes de confiabilidad Alpha de Cronbach de moderados a moderados altos (ver Tabla 1).

Para la sección 2 cuya forma de respuesta era distinta, el análisis factorial arrojó un Índice $\mathrm{KMO}=.638$ y la Pruea de Esfericidad de Bartlett= 453.788, $\mathrm{gl}=6, p=.000$. Puso en evidencia un único factor con valor superior a 1 que explica el $55.21 \%$ de la varianza y un coeficiente de confiabilidad Alpha de Cronbach moderado (ver Tabla 2).

Tabla 2. Análisis factorial de la Sección 2 de la Encuesta de Salud SF-36, Ciudad de México

\begin{tabular}{lc}
\hline Salud General & $\alpha=.73$ \\
\hline Creo que mi salud va a empeorar. & .782 \\
Estoy tan sano como cualquiera. & .762 \\
Mi salud es excelente. & .737 \\
Creo que me pongo enfermo más & .688 \\
fácilmente que otras personas. & \\
\multicolumn{1}{c}{ Media= } & \\
\hline
\end{tabular}

\section{Ciudad de Pachuca, Hidalgo}

Estos resultados permiten observar que 42 de los 45 ítems diseñados cumplieron con los criterios establecidos por Reyes Lagunes, García y Barragán (2008). Acto seguido, los reactivos de la sección 1 se analizaron por medio de un análisis factorial de componentes principales con rotación ortogonal (tipo varimax) y se seleccionaron sólo aquellos que registraron cargas factoriales iguales o superiores a $.40 . \mathrm{El}$ análisis factorial arrojó un índice $\mathrm{KMO}=.683$ y la Prueba de Esfericidad de Bartlett=13419.338, gl=780, $p=000$. A pesar de indicar la existencia de 8 factores, debido a la incongruencia en 3 factores, se decidió tomar en cuenta solo 5 factores con valores superiores a 1 que explicaron el $72.99 \%$ de la varianza y con coeficientes de confiabilidad Alpha de Cronbach de moderados altos a altos (ver Tabla 3).

Para la sección 2 el análisis factorial arrojó un índice $\mathrm{KMO}=.709$ y la Prueba de Esfericidad de Bartlett=433.118, $\mathrm{gl}=6, p=.000$. El mismo puso en evidencia un solo factor con valor superior a 1 que explica el $68.78 \%$ de la varianza y con un coeficiente de confiabilidad Alpha de Cronbach moderado alto (ver Tabla 4). 
Tabla 3. Análisis Factorial de la Sección 1 de la Encuesta de Salud SF-36, Ciudad de Pachuca, Hidalgo

\begin{tabular}{|c|c|}
\hline Función Física & $\alpha=.95$ \\
\hline Caminar una sola manzana (unos 100 metros). & .877 \\
\hline Caminar un kilometro o más. & .874 \\
\hline Subir un solo piso por la escalera. & .852 \\
\hline Cargar o llevar bolsa de la compra. & .840 \\
\hline Caminar varias manzanas (varios centenares de metros). & .837 \\
\hline $\begin{array}{l}\text { Hacer esfuerzos moderados, como mover una mesa, pasar la aspiradora, jugar boliche o caminar más de } \\
\text { una hora. }\end{array}$ & 669 \\
\hline Hacer esfuerzos intensos, tales como correr, levantar objetos pesados, o participar deportes agotadores. & .545 \\
\hline Realiza sus actividades habituales aunque sienta dolor. & .429 \\
\hline \multicolumn{2}{|l|}{ Media=2.065; DS=1.0143 } \\
\hline ¿Hizo menos de lo que hubiera querido hacer, a causa de su salud física? & .870 \\
\hline A causa de su salud física ¿dejó de hacer por completo sus actividades cotidianas o trabajo? & .843 \\
\hline $\begin{array}{l}\text { ¿Tuvo alguna dificultad para hacer su trabajo o sus actividades cotidianas (por ejemplo, le costó más de lo } \\
\text { normal), a causa de su salud física? }\end{array}$ & .783 \\
\hline $\begin{array}{l}\text { ¿Hasta qué punto sus problemas emocionales han dificultado sus actividades sociales habituales con la } \\
\text { familia, los amigos, los vecinos u otras personas? }\end{array}$ & .572 \\
\hline $\begin{array}{l}\text { ¿Hasta qué punto su salud física ha dificultado sus actividades sociales habituales con la familia, los } \\
\text { amigos, los vecinos u otras personas? }\end{array}$ & .476 \\
\hline
\end{tabular}
amigos, los vecinos $\mathrm{u}$ otras personas? $\quad$ Media=1.838; DS=.9196

\begin{tabular}{|c|c|}
\hline Rol Emocional & $\alpha=.94$ \\
\hline $\begin{array}{l}\text { ¿Puso menos atención a la hora de estar realizando su trabajo o actividades cotidianas debido a algún } \\
\text { problema emocional (como estar triste, deprimido o nervioso)?. }\end{array}$ & .760 \\
\hline $\begin{array}{l}\text { ¿Mostró menos interés en convivir con otras personas debido a algún problema emocional (como estar } \\
\text { triste, deprimido o nervioso)?. }\end{array}$ & .739 \\
\hline $\begin{array}{l}\text { ¿Hizo menos de lo hubiera querido hacer, a causa de algún problema emocional (como estar triste, } \\
\text { deprimido o nervioso)? }\end{array}$ & .713 \\
\hline $\begin{array}{l}\text { ¿Tuvo que reducir el tiempo dedicado a su trabajo o a sus actividades cotidianas a causa de algún } \\
\text { problema emocional (como estar triste, deprimido o nervioso)?. }\end{array}$ & .546 \\
\hline \multicolumn{2}{|l|}{ Media $=1.904$ DS $=.9232$} \\
\hline Vitalidad & $\alpha=.86$ \\
\hline ¿Qué tanto se sintió feliz? & .672 \\
\hline $\begin{array}{l}\text { ¿Qué tanto su salud física le ha dificultado realizar sus actividades sociales (como visitar a los amigos o } \\
\text { familiares)?. }\end{array}$ & .651 \\
\hline ¿Qué tanto se sintió débil? & .511 \\
\hline ¿Qué tanto se sintió desanimado y triste? & .475 \\
\hline \multicolumn{2}{|l|}{ Media $=2.625 \mathrm{DS}=.4679$} \\
\hline Dolor Corporal & $\alpha=.91$ \\
\hline ¿El dolor físico que ha tenido le ha generado algún problema emocional? & .726 \\
\hline $\begin{array}{l}\text { ¿Hasta qué punto el dolor le ha dificultado su trabajo habitual (incluido el trabajo fuera de casa y las tareas } \\
\text { doméstica?. }\end{array}$ & .671 \\
\hline $\begin{array}{l}\text { Hasta qué punto el dolor le ha impedido realizar su trabajo habitual (incluido el trabajo fuera de casa y las } \\
\text { tareas doméstica? }\end{array}$ & 669 \\
\hline
\end{tabular}

Tabla 4. Análisis Factorial de la Sección 2 de la Encuesta de Salud SF-36, Ciudad de Pachuca, Hidalgo

\begin{tabular}{lc}
\hline Salud General & $\alpha=.85$ \\
\hline Mi salud es excelente. & .889 \\
Creo que mi salud va a empeorar. & .820 \\
Estoy tan sano como cualquiera. & .816 \\
Creo que me pongo enfermo más fácilmente & .789 \\
que otras personas. &
\end{tabular}

Media $=3.587 ; \mathrm{DS}=.9073$ 
Tabla 5. Análisis Factorial de la Sección 1 de la Encuesta de Salud SF-36, Ciudad de Gómez Palacio, Durango

\begin{tabular}{|c|c|}
\hline Función Física & $\alpha=.94$ \\
\hline Caminar una sola manzana (unos 100 metros). & .861 \\
\hline Subir un solo piso por la escalera. & .852 \\
\hline Caminar varias manzanas (varios centenares de metros). & .833 \\
\hline Bañarse o vestirse por si mismo. & .804 \\
\hline Agacharse o arrodillarse. & .795 \\
\hline Hacer esfuerzos moderados, como mover una mesa, pasar la aspiradora, jugar boliche o caminar mas de una hora. & .785 \\
\hline Caminar un kilometro. & .765 \\
\hline Cargar o llevar bolsa de la compra. & .750 \\
\hline Subir varios pisos por la escalera. & .746 \\
\hline Media $=1.79 ; \mathrm{DS}=.9528$ & \\
\hline
\end{tabular}

¿Tuvo que dejar de hacer algunas tareas en su trabajo o en sus actividades cotidianas, a causa de sus salud fisica?.

A causa de su salud fisica ¿dejó de hacer por completo sus actividades cotidianas o trabajo?.

¿Tuvo que reducir el tiempo dedicado al trabajo o a sus actividades cotidianas a causa de su salud fisica?.

¿Tuvo alguna dificultad para hacer su trabajo o sus actividades cotidianas(por ejemplo, le costó más de lo normal), a causa .774 de su salud fisica?

¿Hasta que punto el dolor le ha dificultado su trabajo habitual (incluido el trabajo fuera de casa y las tareas domésticas?. .670

¿Hizo menos de lo que hubiera querido hacer, a causa de su salud fisica?.

Hasta que punto el dolor le ha impedido realizar su trabajo habitual (incluido el trabajo fuera de casa y las tareas doméstica?. .521

¿Hasta que punto su salud fisica ha dificualtado sus actividades sociales habituale con la familia, los amigos, los vecinos u .459 otras personas?.

Media=1.59; DS=.8380

Rol Emocional

¿Puso menos atencion a la hora de estar realizando su trabajo o actividades cotidianas debido a algún problema emocional .766 (como estar triste, deprimido o nervioso)?

¿Mostró menos interes en convivir con otras personas debido a algún problema emocional (como estar triste, deprimido o .765 nervioso)?.

¿Hizo menos de lo hubiera querido hacer, a causa de algun problema emocional (como estar triste, deprimido o nervioso)?. .745

¿No hizo su trabajo o sus actividades cotidianas tan cuidadosamente como de costumbre a causa de algùn problema .737 emocional (como estar triste, deprimido o nervioso)?.

¿Tuvo que reducir el tiempo dedicado a su trabajo o a sus actividades cotidianas a causa de algùn problema emocional .661 (como estar triste, deprimido o nervioso)?.

¿Hasta que punto sus problemas emocionales han dificultado sus actividades sociales habituales con la familia, los amigos, .613 los vecinos u otras personas?

¿Qué tanto estuvo muy nervioso?

¿Qué tanto se sintio tan bajo de moral que nada podía animarle?

¿El dolor fisico que ha tenido le ha generado algún problema emocional?

Media $=1.73 ; \mathrm{DS}=.8259$

\begin{tabular}{cc}
\hline Función Social y Vitalidad & $\alpha=.75$ \\
\hline ¿Qué tanto su salud fisica le ha dificultado realizar sus actividades sociales (como visitar alos amigos o familiares)?. & .736
\end{tabular}

¿Qué tanto se sintió cansado?.

Media=2.03; DS=.7943

Vitalidad y Dolor Corpora

\section{Ciudad Gómez Palacio, Durango}

Se observó en este caso que 42 de los 45 ítems diseñados cumplieron con los criterios establecidos por Reyes Lagunes, García y Barragán (2008). Acto seguido, se analizaron los reactivos de la sección 1 por medio de un análisis factorial de componentes principales con rotación ortogonal (tipo varimax) y se seleccionaron sólo aquellos que registraron cargas factoriales iguales o superiores a .40 . El análisis factorial de componentes principales arrojó un índice 
KMO=.909 y la Prueba de Esfericidad de Bartlett=7231.292, $\mathrm{gl}=595, \quad p=.000$. Además indicaron la existencia de 5 factores con valores superiores a 1 que explicaron el $68.103 \%$ de la varianza y coeficientes de confiabilidad Alpha de Cronbach de moderados a altos (ver Tabla 5).

Para la sección 2 cuya forma de respuesta era distinta, el análisis factorial arrojó un índice $\mathrm{KMO}=.688$ y la Prueba de Esfericidad de Bartlett $=216.029, \mathrm{gl}=6, p=.000$ ). Además indicó su existencia con valor superior a 1 que explica el $56.24 \%$ de la varianza y un coeficiente de confiabilidad Alpha de Cronbach moderado (ver Tabla 6).

Tabla 6. Análisis Factorial de la Sección 2 de la Encuesta de Salud SF-36, Ciudad de Gómez

Palacio, Durango

\begin{tabular}{lc}
\hline \multicolumn{1}{c}{ Salud General } & $\alpha=.73$ \\
\hline Creo que mi salud va a empeorar. & .788 \\
Estoy tan sano como cualquiera. & .777 \\
Mi salud es excelente. & .770 \\
Creo que me pongo enfermo más & .658 \\
fácilmente que otras personas. & \\
$\quad$ Media=3.73; DS=.9109 & \\
\hline
\end{tabular}

\section{Discusión}

El propósito de esta investigación fue la validación en México en tres contextos culturales distintos (Ciudad de México, Ciudad de Pachuca, Hidalgo y Ciudad de Gómez Palacio, Durango) de la medida SF-36 y la identificación de las similitudes y/o diferencias en su configuración factorial. Para ello se siguió el procedimiento de Reyes Lagunes, García y Barragán (2008) encaminado a obtener medidas válidas y culturalmente sensibles para la población mexicana seleccionada. Luego de esto, se obtuvieron configuraciones factoriales similares, pero con diferencias.

Inicialmente hay que señalar que tanto el reactivo de transición de salud cuya formulación corrsponde a "¿Cómo diría que es su salud comparada con la de hace un año?", y el que interroga sobre "¿Qué tan mala o buena es su salud?" dadas su formas de respuestas (de "Mucho peor ahora que hace un año" a "Mucho mejor ahora que hace un año" y de "Mala" a "Muy buena") se dejaron como indicadores y no se obtuvo su confiabilidad. Por su parte, la
Sección 1 cuyos estímulos son "¿Qué tanto su salud actual le limita para..." y "Durante las últimas cuatro semanas...", se analizaron en forma conjunta en un solo análisis factorial, debido a que poseían el mismo formato de respuesta (Nada a Mucho) La Sección 2, cuya forma de respuesta podía variar de "Totalmente falsa" a "Totalmente cierta", fue analizada separadamente en otro análisis factorial. En cuanto a las dimensiones evaluadas por el instrumento en cuestión se pueden mencionar: función física, rol físico, dolor corporal, salud general, vitalidad, función social, rol emocional, salud mental y el ítem de transición de salud.

Cabe señalar que en la versión original (usada para una muestra en el sureste por Zúñiga et al., 1999) cada dimensión tiene entre 1 y 10 reactivos, de modo tal que aparece cierto desequilibrio en la operacionalización de cada dimensión. Esta circunstancia trató de resolverse al agregarse algunos otros reactivos que enriquecieran el instrumento. En la Tabla 7 que se presenta a continuación, se encuentran las dimensiones originales y las obtenidas en la ciudadades de México; Pachuca, Hidalgo y Gómez Palacio, Durango. Asimismo aparecen los coeficientes de confiabilidad reportados por Vilagut et al., (2005) luego de realizar un meta-análisis de la investigación llevada a cabo con el SF-36 en una década y aquellos obtenidos en esta investigación. Todo esto con el fin de comparar sus configuraciones factoriales y sus características psicométricas de validez y confiabilidad.

Como se observa, los factores de función física y salud general se mantuvieron exactamente iguales a los de la versión original, lo cual les da una validez de constructo robusta. En cuanto a sus coeficientes de confiabilidad alpha de Cronbach, el estudio de Vilagut et al., (2005) reporta

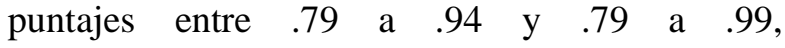
respectivamente, mientras que para las tres muestras de éste estudio se observaron puntajes entre .94 a .95 y .73 a .85 . En función de ello, estos factores quedaron definidos de la siguiente manera:

Función Física. Limitaciones para realizar actividades fisicas como correr, levantar objetos pesados, caminar a lo largo de varias manzanas, cargar bolsas, subir escaleras, realizar quehaceres 
Tabla 7. Comparación de los factores originales y los obtenidos en la presente investigación

\begin{tabular}{|c|c|c|c|c|c|c|c|}
\hline Original & $\alpha$ & México & $\alpha$ & Pachuca & $\alpha$ & Gómez Palacio & $\alpha$ \\
\hline Función física & $.79-.94$ & Función física & .94 & Función física & .95 & Función física & .94 \\
\hline Rol físico & $.79-.99$ & Rol físico & .95 & Rol físico & .94 & $\begin{array}{l}\text { Rol fisico y } \\
\text { Dolor corporal }\end{array}$ & .94 \\
\hline $\begin{array}{l}\text { Dolor } \\
\text { corporal }\end{array}$ & $.65-.92$ & Dolor corporal & .83 & Dolor corporal & .91 & - & \\
\hline Vitalidad & $.70-.86$ & Vitalidad & .76 & Vitalidad & .86 & $\begin{array}{c}\text { Vitalidad y } \\
\text { Dolor corporal }\end{array}$ & .68 \\
\hline $\begin{array}{l}\text { Función } \\
\text { social }\end{array}$ & $.45-.81$ & - & & - & - & $\begin{array}{c}\text { Función social } \\
\text { y Vitalidad }\end{array}$ & .75 \\
\hline $\begin{array}{l}\text { Rol } \\
\text { emocional }\end{array}$ & $.58-.94$ & $\begin{array}{l}\text { Rol emocional } \\
\text { y Función } \\
\text { social }\end{array}$ & .93 & Rol emocional & .94 & Rol emocional & .92 \\
\hline Salud mental & $.76-.85$ & - & & - & & - & \\
\hline $\begin{array}{l}\text { Salud general } \\
\text { Ítem de }\end{array}$ & $.71-.85$ & Salud general & .73 & Salud general & .85 & Salud general & .73 \\
\hline $\begin{array}{l}\text { Transición de } \\
\text { salud }\end{array}$ & & Indicador & & Indicador & & Indicador & \\
\hline
\end{tabular}

de la casa o incluso bañarse o vestirse debido a la salud física actual.

Salud General. Estimación de la salud como excelente, como la de cualquiera, que va a empeorar o bien que la enfermedad aparece más facilmente que en otras personas.

En cuanto al Rol Físico y Vitalidad se encontró que para la Ciudad de México y para la Ciudad de Pachuca la configuración fue igual a la original. En cambio, en la Ciudad de Gómez Palacio, se mezcló con el dolor corporal, lo cual resulta entendible ya que el individuo puede dejar de funcionar apropiadamente por cuestiones de salud, incluido el dolor que puede acompañarlas. Por su parte, la dimensión de rol emocional mostró similitudes entre la Ciudad de Pachuca y la de Gómez Palacio. En la Ciudad de México se combinó con la función social, situación explicable,al igual que en el caso anterior, ya que el individuo puede no funcionar debidamente en sus esferas sociales y laborales debido a la experiencia emocional negativa que puede

bloquear su desenvolvimiento óptimo. En lo que toca a sus coeficientes de confiabilidad se puede notar que en la versión original oscilaron entre .79 y .99 para el rol físico, entre .70 a .86 para la vitalidad y entre .58 a .94 para el rol social, mientras que en la presente investigación se obtuvieron coeficientes entre .94 a $.95, .68$ a .76 y .92 a .94 respectivamente. Esto indica que para el rol físico y para el rol social los datos obtenidos son en general superiores a lo observado en el estudio de Vilagut et al., (2005) y que en el caso de vitalidad los coeficientes obtenidos fueron menores en comparación con dicho estudio. Tomando en cuenta todas las cuestiones mencionadas, los factores quedaron definidos como se detalla a continuación.

Rol Físico. Dificultades pare realizar trabajo, actividades cotidianas, tareas domésticas dentro de la casa e incluso aquellas sociales debido a la salud física.

Rol Físico y Dolor Corporal. Dificultades pare realizar trabajos, actividades cotidianas, tareas domésticas dentro de la casa e incluso las sociales debido al dolor y a la salud física.

Vitalidad. Energia, vitalidad y felicidad que tiene el individuo o por lo contrario calma $y$ tranquilidad.

Vitalidad y Dolor Corporal. Energia, vitalidad y felicidad que tiene el individuo o bien calma y tranquilidad aún cuando se experimenta dolor.

Rol Emocional. Problemas emocionales, como estar triste, deprimido o nervioso, que afectan el grado de atención, el interés en convivir con otras personas, sentir decaimiento moral, hacer las actividades cotidianas o reducir el tiempo dedicado al trabajo.

Rol Emocional y Función Social. Sentimientos de tristeza, depresión, nerviosismo, o desánimo que dificultan la atención, el trabajo, las actividades diarias y el interés en convivir con otras personas, tales como familiares, amigos, vecinos u otras. 
Con respecto al dolor corporal, que en la versión original presentaron coeficientes de confiabilidad de entre .65 a .92 , en esta investigación oscilaron entre $\quad .83$ y $\quad .91 \quad$ y únicamente se obtuvieron puros en las ciudadades de México y de Pachuca. En el caso de la Ciudad de Durango los reactivos se acomodaron junto con el factor de rol físico y con el factor de vitalidad. En consecuencia, este factor quedo conceptuado de la siguiente manera.

Dolor Corporal. Monto de dolor en alguna parte del cuerpo, su duración, su vinculación con algún problema emocional $\mathrm{y}$, si aún con dolor, se realizan actividades, se siente cansancio, debilidad o agotamiento.

El factor de función social solo apareció en la muestra de la Ciudad de Gómez Palacio pero en combinación con el de vitalidad y -como ya se mencionó- en la Ciudad de México con el del rol emocional. Esta dimensión originalmente mostró coeficientes de confiabilidad entre .45 a .81 , en la ciudad de Gómez Palacio se obtuvo uno de .75 y conjuntamente con el rol emocional de .93. En función de ello se define su combinación de la siguientes forma:

Función Social en combinación con Vitalidad: Monto de dolor en alguna parte del cuerpo y su efecto en las actividades sociales, en el cansancio, el desánimo, la tristeza y la debilidad.

Respecto del factor relativo a la salud mental, no surgió claramente en los análisis de validación. Sus reactivos -5 en la versión inicial- quedaron distribuidos en los otros factores sin una marcada presencia en ninguno de ellos. Esto puede deberse a que al evaluar angustia, depresión, felicidad, tranquilidad y calma, se fusionaran con otros aspectos de la salud que tenían que ver con emociones y con las relaciones personales $\mathrm{o}$ sociales del individuo, que - por su naturaleza involucran la experiencia emocional.

Es claro hasta este punto que se encontraron variaciones interesantes entre la versión originaria y lo obtenido en las tres sedes estudiadas en esta pesquisa. Esto puede obedecer a que en las otras muestras con las cuales se trabajó o aplicó esta escala en distintas investigaciones, se incluyeron pacientes con cáncer (Zhou et al., 2011; Shields, Travis, \& Rousseau, 2010; Harkness Hodgson, Shields, \& Rousseau, 2003; Galbraith, Pedro, Jaffe, \& Allen, 2008), diabetes (Trief, Orendorff,
Himes, \& Weinstock, 2001), personas con problemas psicológicos tras un divorcio (Butterworth \& Rodgers, 2008), esclerosis múltiple (Janssens et al., 2003), infertilidad (Nelson, Shindel, Naughton, Ohebshalom, \& Mulhall, 2008) y de abuso sexual (Hensing \& Alexanderson, 2000). Contrariamente los participantes de la presente investigación fueron adultos mexicanos de tres ciudades ubicadas geográficamente en lugares distintos, presumiblemente sanos, con pareja y con escolaridades principalmente de preparatoria y de licenciatura. Además las muestras provenían de nichos socio-culturales distintos a los correspondientes a la validación inicial: una corresponde a un muestra estadounidense y en otra se trabajó por díez años en España (Vilagut et al., 2005). Aunque se encontró un trabajo realizado en el sureste de México, la muestra fue menor a las estudiadas aquí y se desconocen datos específicos más allá de la ausencia de enfermedades crónicas y del trabajo en el gobierno (Zúñiga et al., 1999). Además de la procedencia, sus características y la época en la que se realizaron dichos estudios, constituyen aspectos centrales en la conformación de los factores de la escala en las sedes, al igual que la variabilidad en sus coeficientes de confiabilidad.

Estos hallazgos representan una aproximación al perfeccionamiento de la medición de la salud física y mental en población mexicana y se seguirá trabajando no solo para conocer divergencias entre sexos, nivel de esolaridad o grupo socio-cultural, sino también en conjunción con otras variables de salud, como resiliencia, regulación emocional, intimidad, pasión y empatía en las relaciones románticas.

\section{Referencias}

Ardila R. (2003). Calidad de Vida: Una definición integradora. Revista Latinoamericana, 35(2), 161-164.

Butterworth, P., \& Rodgers, B. (2008). Mental health problems and marital disruption: Is it the combination of husbands and wives' mental health problems that predicts later divorce? Social Psychiatry Psychiatrician Epidemiology, 43, 758-763. 
Cruceanu, D. Muntele, I., \& Cozma, D. (2014). Health-an autoevaluation Predictor of Personal life quality. Cross-Cultural Management Journal, XVI(5), 67-78.

Fleuret, S., \& Thouez, J. P. J.(2007). Géographie de la santé, un panorama. Angers: Editions Economica-Anthropos.

Galbraith, M. E., Pedro, L. W., Jaffe, A. R., \& Allen, T. L. (2008). Describing health realated outcomes for couples experiencing prostate cancer: Differences and similarities. Oncology Nursing Forum, 35(5), 794-801.

Guyatt, G. H., Kirshner, B., \& Jaeschke, R. (1992). Measuring health status: What are the necessary measurement properties? Journal of Clinical Epidemiology, 45(2), 1341-1345.

Hensing, G., \& Alexanderson, K. (2000). The relation of adult experience of domestic harassment, violence, and sexual abuse to health and sickness absence. International Journal of Behavioral Medicina, 7(1), 1-18.

Hodgson, J. H., Shields, C. G., \& Rousseau, S. L. (2003). Disengaging communication in laterlife couples coping with breast cancer. Families, Systems \& Health, 21(2), 145-163.

Janssens, A. C. J. W., van Doorn, P. A., de Boer, J. B., van der Meché, F. G. A., Passchier, J., \& Hintzen, R. Q. (2003). Impact of recently diagnosed multiple sclerosis on quality of life, anxiety, depression and distress of patients and partners. Acta Neurológica Scandinavica, 108, 389-395.

Kerlinger, F. N. \& Lee, H. B. (2002). Investigación del Comportamiento. México: McGraw-Hill

Nelson, C. J., Shindel, A. W., Naughton, C. K., Ohebshalom, M., \& Mulhall, J. P. (2008). Prevalence and predictors of sexual problms, relationship stress, and depression in female partners of infertile couples. Journal of Sexual Medicine, 5, 1907-1914.

Reyes-Lagunes, I., \& García, \& Barragán, L. F. (2008). Hacia un procedimiento de validación psicométrica culturalmente relevante. En S. Rivera-Aragón, R. Díaz-Loving, R. SánchezAragón y I. Reyes-Lagunes (Eds.), La Psicología Social en México XII (pp. 625630). México: AMEPSO.

Shields, C. G., Travis, L. A., \& Rosseau, 1. (2010). Marital attachment and adjustment in older couples coping with cancer. Anging Mental Health, 4(3), 223-233.

Shindel, A. W., Nelson, C. J., Naughton, C. K., Ohebshalom, M., \& Mulhalla, J. P. (2008). Sexual function and quality of life in the male partner of infertile couples: Prevalence and correlates of dysfunction. The Journal of Urology, 179, 1056-1069.

Shmueli A. (2002). Reporting heterogeneity in the measurement of health and health-related quality of life. Pharmacoeconomics, 20(6),405-412.

Trief, P. M., Orendorff, R., Himes, C. L., \& Weinstock, R. S. (2001). The marital relationships and psychosocial adaptation and glycemic control of individuals with diabetes. Diabetes Care, 24(8), 1384-1389.

Vilagut, G. Ferrer, M. Rebollo, P. Permanyer, G. Quintana, J. Santed, R. Valderas, J. Ribera, A. Domingo, A., \& Alonso, J. (2005). El Cuestionario de Salud SF-36 español: Una década de experiencia y nuevos desarrollos. Gaceta Sanitaria, 19(2), 135-150.

Zhou, E. S., Kim, Y., Rasheed, M., Benedict, C., Bustillo, N. E., Soloway, M., Kava, B. R., \& Penedo, F. J. (2011). Marital satisfaction of advanced prostate cancer survivors and their spousal caregivers: The dyadic effects of physical and mental health. Psycho-Oncology, 20, 1353-1357.

Zúñiga, M. A., Carrillo-Jiménez, G. T., Fos, P. J., Gandek, B., \& Medina-Moreno, M. R. (1999). Evaluación del estado de salud con la Encuesta SF-36: Resultados preliminares en México. Salud Pública de México, 41(2), 110118. 